\title{
Anti-apoptotic Effects of House Dust Mite, S100A8 and S100A9 on Spontaneous Apoptosis of Neutrophils in Coculture with Immune Cells and in the Presence of T Helper Cytokines
}

\author{
In Sik Kim ${ }^{1,2}$ and Ji-Sook Lee $\mathrm{L}^{3, \dagger}$ \\ ${ }^{1}$ Department of Biomedical Laboratory Science, School of Medicine, Eulji University, Daejeon 301-746, Korea \\ ${ }^{2}$ Department of Senior Healthcare, BK21 Plus Program, Graduate School, Eulji University, Daejeon 301-746, Korea \\ ${ }^{3}$ Department of Clinical Laboratory Science, Wonkwang Health Science University, Iksan 570-750, Korea
}

House dust mite (HDM) as a major allergen and damage-associated molecular pattern (DAMP) such as S100A8 and S100A9 trigger the pathogenesis and severity of allergic disease such as asthma. Regulation of neutrophil apoptosis is an important immune response and its dysregulation is involved in pathogenesis of allergic diseases. In this study, we examined the effects of HDM, S100A8 and S100A9 on spontaneous apoptosis of normal neutrophils. We considered the importance of the difference between in vitro and in vivo results and developed a new in vitro system consisting of a combination of immune cells and T helper (Th) cytokines. Extract of Dermatophagoides pteronyssinus (DP), S100A8, and S100A9 inhibited neutrophil apoptosis in culture of neutrophils alone without other leukocytes. DP and S100A8 more strongly suppressed neutrophil apoptosis in combinations of neutrophils, eosinophils, lymphocytes or monocytes than in a culture of neutrophils alone. Anti-apoptotic effect of S100A9 in the mixture of immune cells was similar to that in neutrophils. DP, S100A8, and S100A9 blocked neutrophil apoptosis, regardless of pretreatment with a T helper (Th) 1 cytokine (IFN- $\gamma$ ), Th2 cytokines (IL-4 and IL-10), a Th9 cytokine (IL-9), a Th17 cytokine (IL-17), a Treg-producing cytokine (TGF- $\beta$ ). These findings may enable elucidation of allergy pathogenesis due to HDM and DAMP.

Key Words: House dust mite, S100A8, S100A9, Neutrophil apoptosis

Allergic diseases include asthma, allergic rhinitis, and atopic dermatitis depending on a target organ. House dust mite (HDM) is an essential allergen of allergic diseases (Gaffin and Phipatanakul, 2009; Kemp, 2009; Leung and Guttman-Yassky, 2014). HDM allergens contain Dermatophagoides pteronyssinus (DP) and Dermatophagoides farina (DF), which affect pathogenesis of allergic diseases (Milián and Diaz, 2004; Kang et al., 2014). Damage-associated

\footnotetext{
* Received: April 22, 2015 / Revised: June 6, 2015

Accepted: June 8, 2015

${ }^{\dagger}$ Corresponding author: Ji-Sook Lee. 501, Iksandaero, Department of Clinical Laboratory Science, Wonkwang Health Science University, Iksan 570-750, Korea.

Tel: +82-63-840-1216, Fax: +82-63-840-1219

e-mail: jslee1216@wu.ac.kr

(C)The Korean Society for Biomedical Laboratory Sciences. All rights reserved.
}

molecular pattern (DAMP) is associated with immune response in the noninfectious inflammation such as allergy and includes S100A8 and S100A9. Both S100A8 and S100A9 belong to the S100 protein family and are involved in allergic disease, cancer and autoimmune disease (Donato et al., 2013; Kim et al., 2013). Neutrophils function as inflammatory cells. Inhibition of constitutive neutrophil apoptosis aggravates pathogenic lesions via continuous accumulation of neutrophils in allergic diseases such as asthma (ScheelToellner et al., 2004; Monteseirín, 2009; Lee et al., 2014).

This study was approved by the Institutional Review Board of Eulji University for normal volunteers. All participants in this study gave their written informed consent. Recombinant S100A8 and S100A9 proteins were produced 
as follows. Total RNA of human neutrophils was extracted using TRIzol reagent (Life Technologies Inc.) and first strand cDNA was synthesized with AccuPower RT PreMix

(Bioneer, Daejeon, Korea). Primers S100A8-1 (5'-ttccatat-

A

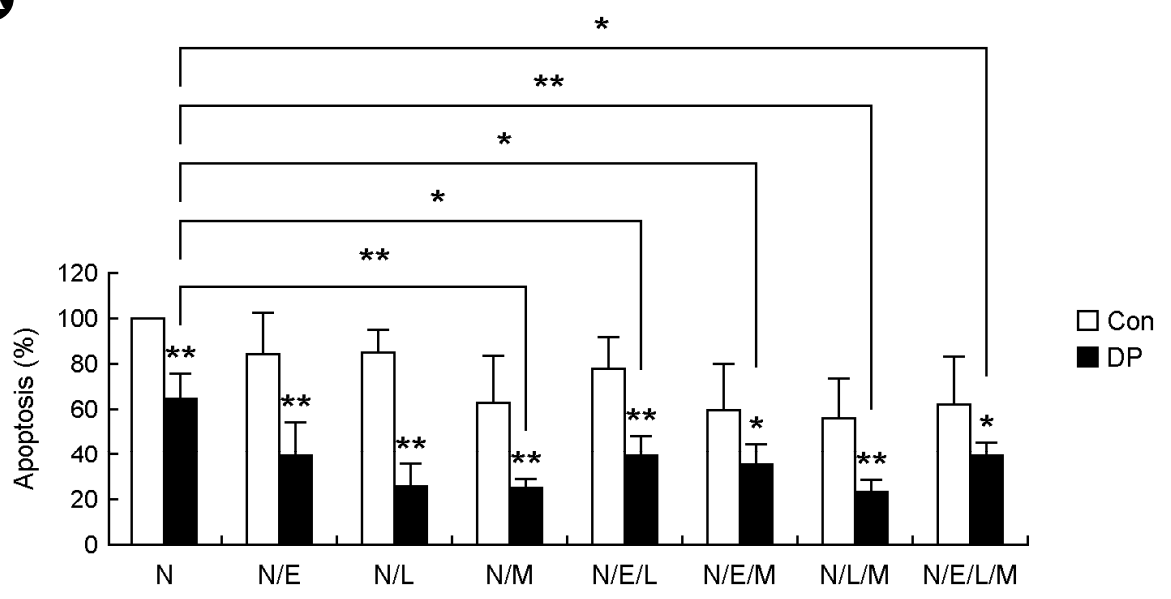

B

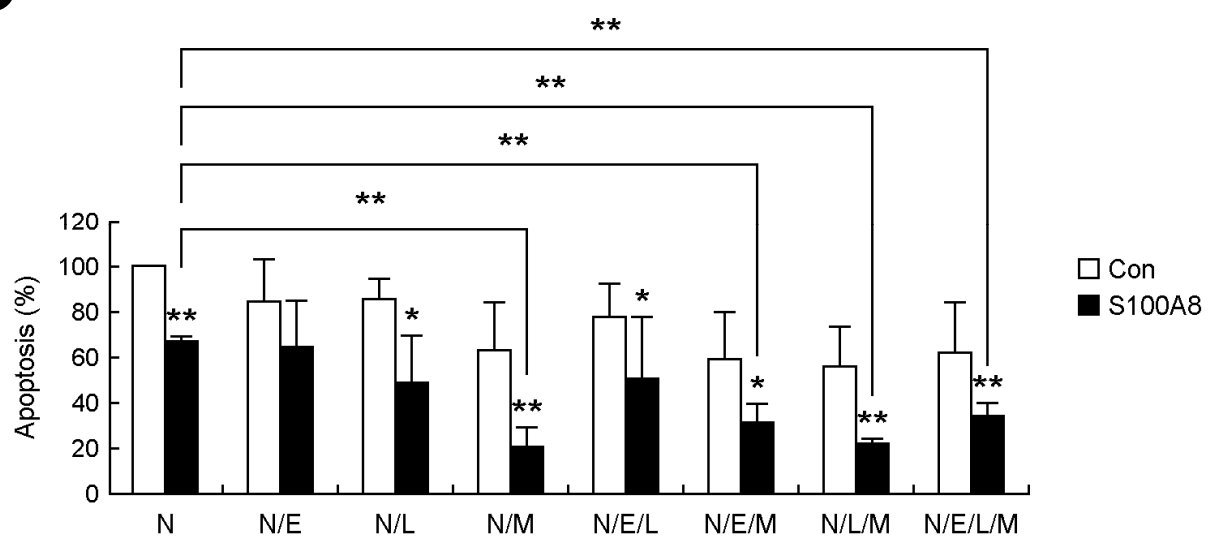

C

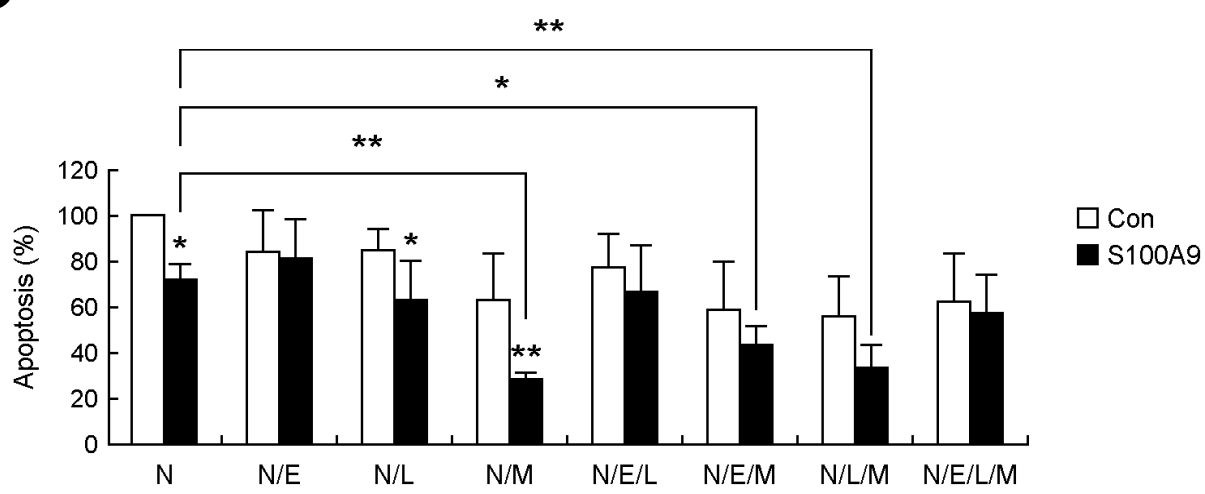

Fig. 1. DP, S100A8, and S100A9 have anti-apoptotic effects on neutrophils in coculture with immune cells. (A) Normal neutrophils were isolated from the peripheral blood of normal subjects $(3<\mathrm{n}<8)$. The neutrophils $(\mathrm{N})$ were incubated for $24 \mathrm{~h}$ in the absence and presence of eosinophils (E), lymphocytes (L) or monocytes (M) with or without DP, S100A8, and S100A9 $(10 \mu \mathrm{g} / \mathrm{mL})$. Apoptosis was analyzed by measuring the binding of annexin V-FITC and PI. Data are expressed as the means means \pm SD and are presented relative to the control, which was set at $100 \%$. $* P<0.05$ and $* * P<0.01$ indicate a significant difference between the untreated control and DP, S100A8, or S100A9-treated groups. 
gatgttgaccgagctggagaa), S100A8-2 (5'-ccgctcgagctactctttgtggctttctt), S100A9-1 (5'-ttccatatgatgacttgcaaaatgtcgca), and S100A9-2 (5'-ccgctcgagactgtggtcttagggggtgc) were used for cDNA synthesis of S100A8 and S100A9. Double-stranded cDNA of human S100A8 and S100A9 was synthesized using polymerase chain reaction $\left(94^{\circ} \mathrm{C} / 30 \mathrm{sec}, 60^{\circ} \mathrm{C} / 30 \mathrm{sec}\right.$, $72^{\circ} \mathrm{C} / 50 \mathrm{sec}, 30$ cycles) and subsequently cloned into pET28 expression vector (Merck Millipore, Darmstadt, Germany). Recombinant S100A8 and S100A9 expressions were induced with $1 \mathrm{mM}$ isopropyl $\beta$-D-thiogalactoside in $E$. coli BL21 (DE3, Merck Millipore) for $4 \mathrm{~h}$ and $16 \mathrm{~h}$ at $37^{\circ} \mathrm{C}$, respectively. Thereafter, the bacteria were centrifuged at 5,000 g for $10 \mathrm{~min}$ and the pellet was lysed in BugBuster Protein Extraction reagent (Merck Millipore). Next, the lysate was centrifuged at $55,000 \mathrm{~g}$ for $30 \mathrm{~min}$ at $4{ }^{\circ} \mathrm{C}$, after which the supernatant was collected. Recombinant His-Tag
S100A8 and S100A9 were purified using a nickel column and the purified proteins were verified by SDS-PAGE and western blotting. Human neutrophils were isolated from the heparinized peripheral blood of healthy persons using FicollHypaque gradient centrifugation and a CD16 microbeads magnetic cell sorting kit (Miltenyi Biotec, Bergisch Gladbach, Germany). The cells were washed after hypotonic lysis to remove erythrocytes and then resuspended at $3 \times 10^{6} / \mathrm{ml}$ in RPMI 1640 medium with 1\% penicillin-streptomycin and $10 \%$ FBS. An annexin V-fluorescein isothiocyanate (FITC) apoptosis detection kit (BD Biosciences, San Diego, CA, USA) was used to detect neutrophil apoptosis. Isolated neutrophils were incubated with an FITC-labeled annexin $\mathrm{V}$ and propidium iodide (PI) for $15 \mathrm{~min}$ at room temperature. Apoptotic neutrophils were analyzed using a FACSCalibur with CellQuest software (BD bioscience) and were deter-
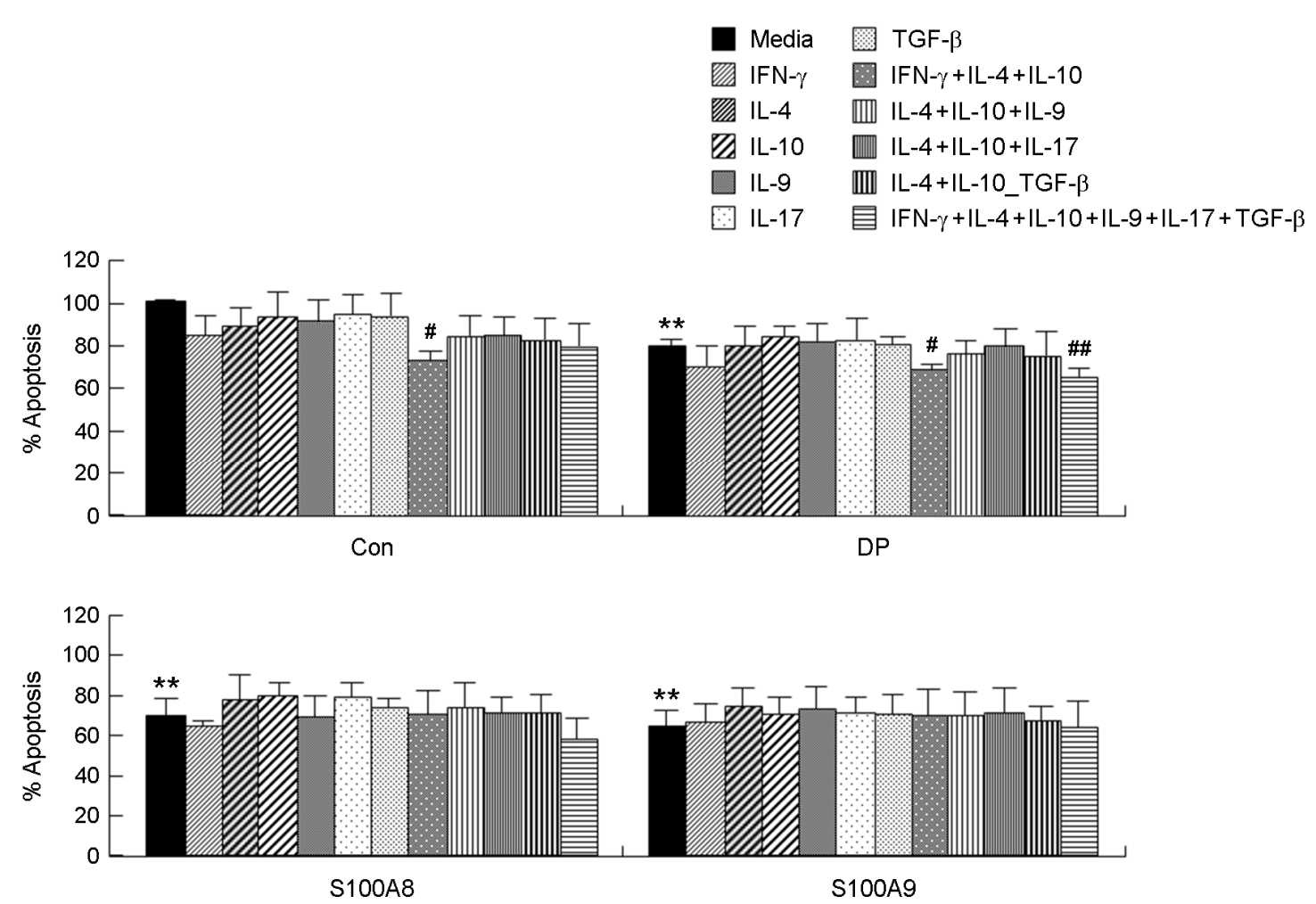

Fig. 2. DP and S100A8 have anti-apoptotic effects on neutrophils in the presence of $\mathbf{T}$ helper cytokines. Normal neutrophils $(\mathrm{n}=3)$ were pretreated for $1 \mathrm{~h}$ in the absence (Con) and presence of IFN- $\gamma(100 \mathrm{ng} / \mathrm{mL}), \mathrm{IL}-4(100 \mathrm{ng} / \mathrm{mL}), \mathrm{IL}-10(100 \mathrm{ng} / \mathrm{mL}), \mathrm{IL}-9(100 \mathrm{ng} / \mathrm{mL})$, IL-17 $(100 \mathrm{ng} / \mathrm{mL})$ and TGF- $\beta(100 \mathrm{ng} / \mathrm{mL})$ and then incubated with or without DP, S100A8, and S100A9 $(10 \mu \mathrm{g} / \mathrm{mL})$. Apoptosis was analyzed by measuring the binding of annexin V-FITC and PI. Data are expressed as the means \pm SD and are presented relative to the control, which was set at $100 \%$. ${ }^{* *} P<0.01$ indicate a significant difference between the untreated control and DP, S100A8, or S100A9-treated groups, and ${ }^{\#} P<0.05$ and ${ }^{\#} P<0.01$ represent a significant difference between the untreated and cytokine-treated groups. 
mined as the percentage of cells showing annexin V+/PIand annexin $\mathrm{V}+/ \mathrm{PI}+$.

Since DP, S100A8, and S100A9 are associated with regulation of neutrophil apoptosis in our previous study (Kim et al., 2015), we examined whether they alter neutrophil apoptosis in new ex vivo environment similar to disease circumstance. Neutrophil apoptosis is not altered by coculture with eosinophils and lymphocytes, except during incubation with monocytes (Fig. 1). DP, S100A8, and S100A9 had inhibitory effects on neutrophil apoptosis in neutrophil alone without other immune cells. DP and S100A8 suppressed neutrophil apoptosis in combinations of neutrophils, eosinophils, lymphocytes or monocytes. S100A9 significantly inhibited neutrophil apoptosis in some combinations such as neutrophils/lymphocytes and neutrophils/ monocytes. S100A8 inhibited similar or stronger neutrophil apoptosis in a mixed culture of immune cells than in a culture of only neutrophils, and the anti-apoptotic effects of S100A9 in the mixture of immune cells is similar to that in neutrophils. Alteration of $\mathrm{T}$ helper (Th) cytokines such as Th1, Th2, Th9, Th17, exist in the lung during asthma progress. We investigated whether DP, S100A8, and S100A9 suppress neutrophil apoptosis in the alteration of Th circumstance. DP, S100A8, and S100A9 blocked neutrophil apoptosis, regardless of pretreatment with a T helper (Th) 1 cytokine (IFN- $\gamma$ ), Th2 cytokines (IL-4 and IL-10), a Th9 cytokine (IL-9), a Th17 cytokine (IL-17), a Treg-producing cytokine (TGF- $\beta$ ) (Fig. 2) or combinations of these cytokines. These results may contribute to a better understanding of the regulation of spontaneous neutrophil apoptosis in normal and asthmatic states.

\section{Acknowledgements}

This paper was supported by Wonkwang Health Science University in 2015.

\section{Conflict of interest}

The authors declare no conflict of interest.

\section{REFERENCES}

Donato R, Cannon BR, Sorci G, Riuzzi F, Hsu K, Weber DJ, Geczy CL. Functions of S100 proteins. Curr Mol Med. 2013. 13: 24-57.

Gaffin JM, Phipatanakul W. The role of indoor allergens in the development of asthma. Curr Opin Allergy Clin Immunol. 2009. 9: 128-135.

Kang BK, Kim MA, Park SH, Lee EJ, Kim JS, Kim EJ, Baek SY, Kim IS. The house dust mite allergen, Dermatophagoides pteronyssinus regulates the constitutive apoptosis and cytokine secretion of human eosinophils. Biomed Sci Lett. 2014. 20: $39-42$.

Kemp AS. Allergic rhinitis. Paediatr Respir Rev. 2009. 10: 63-68. Kim DH, Choi E, Lee JS, Lee NR, Baek SY, Gu A, Kim DH, Kim IS. House dust mite allergen regulates constitutive apoptosis of normal and asthmatic neutrophils via Toll-like receptor 4. 2015. PLoS One (in prsss).

Kim IS, Kim EH, Kim DH, Kim JS, Lee JS. Effect of house dust mite and CCL2 on S100A8 and S100A9 expression in human monocytes. Biomed Sci Lett. 2013. 19: 344-347.

Lee JS, Kim IS. Suppressive effect of arazyme on neutrophil apoptosis in normal and allergic subjects. Biomed Sci Lett. 2014. 20: 244-249.

Leung DY, Guttman-Yassky E. Deciphering the complexities of atopic dermatitis: shifting paradigms in treatment approaches. J Allergy Clin Immunol. 2014. 134: 769-779.

Milián E, Diaz AM. Allergy to House Dust Mites and Asthma. P R Health Sci J. 2004. 23: 47-57.

Monteseirín J. Neutrophils and asthma. J Investig Allergol Clin Immunol. 2009. 19: 340-354.

Scheel-Toellner D, Wang KQ, Webb PR, Wong SH, Craddock R, Assi LK, Salmon M, Lord JM. Early events in spontaneous neutrophil apoptosis. Biochem Soc Trans. 2004. 32: 461-464. 